\title{
A DOMINAÇÃO NO ÂMBITO DAS ORGANIZAÇÕES COM A PERSPECTIVA DE ARTIGOS REFERENCIADOS NA OBRA DE GARETH MORGAN
}

DOMINATION WITHIN ORGANIZATIONS FROM THE PERSPECTIVE OF ARTICLES REFERENCED IN THE WORK OF GARETH MORGAN

\section{Luis Fernando Moreira}

Mestrando em Administração - Inovação e Competitividade pela Universidade de Caxias do Sul (Caxias do Sul/Brasil) E-mail: luismoreiram756yur@gmail.com

Currículo Lattes: http://lattes.cnpq.br/5477750692797803

\section{Daniel Faturi Silva}

Mestrando em Administração - Inovação e Competitividade pela Universidade de Caxias do Sul (Caxias do Sul/Brasil) E-mail: dfsilva20@ucs.br

Currículo Lattes: http://lattes.cnpq.br/0862218255933574

\section{Carlos Eduardo Schlindwein}

Mestrando em Administração - Inovação e Competitividade pela Universidade de Caxias do Sul (Caxias do Sul/Brasil) E-mail: eduschlindwein@hotmail.com

Currículo Lattes: http://lattes.cnpq.br/2487575424708041

\section{Eberson Cordeiro de Almeida}

Especialista em Gestão de Pessoas - Competitividade pelo Centro Universitário Leonardo da Vinci - UNIASSELVI (Novo Hamburgo/Brasil)

E-mail: eber.almeida@hotmail.com

Currículo Lattes: http://lattes.cnpq.br/8732828317802852

Recebido em: 26 de setembro de 2019 Aprovado em: 28 de novembro de 2019 Sistema de Avaliação: Double Blind Review RGD|v. 17 |n. 1 |p. 87-106|jan./abr. 2020 DOI: https://doi.org/10.25112/rgd.v17i1.1843 


\section{RESUMO}

As relações de dominação e trabalho cumprem papeis condescendentes nas organizações, trazendo para os dias atuais a sua essência na gestão contemporânea. Este estudo procurou estudar a teoria da dominação nas organizações, apresentada por meio de metáfora por Gareth Morgan, no livro Imagens da Organização, propondo uma discussão sobre o entendimento do ponto de vista de outros autores. 0 objetivo deste estudo é investigar, através da uma revisão sistemática, de como a dominação está sendo abordada no ponto de vista de autores da atualidade e traçar uma relação com o autor Morgan em uma forma de figura conceitual. A metodologia é de cunho qualitativo descritivo, e estruturada por meio de mineração de dados textuais. Conclui-se que a dominação e a organização têm relação com paradigma do trabalho, e as pessoas que nele estão inseridos continuam subjugadas pelo poder dentro de uma organização, permanecendo e se ampliando em diversos campos, como um paradigma humano no plano organizacional.

Palavras-chave: Dominação. Pessoas. Organizações.

\section{ABSTRACT}

Dominance and work meetings play the patronizing roles in organizations, bringing to the present day their essence in managing things. This study was developed by Gareth Morgan, a group of teaching organizations, with a discussion on the topic of the book view of other authors. The objective of this study is to investigate, through a systematic review, a way that is being approached from the point of view of current authors and a relationship with the author Morgan in a conceptual figure form. The methodology is descriptive qualitative, and is structured through the mining of textual data. It is concluded that domination and information have access with the paradigm of work, and how people who are inserted are continuous subjugated by the power within an organization, remaining and expanding in fields, as a human paradigm without organizational plan.

Keywords: Domination. People. Organizations. 


\section{INTRODUÇÃO}

As ideias existentes sobre poder e controle nos estudos organizacionais podem fornecer um guia útil para responder diversas perguntas. Normalmente as publicações consideram a interação entre quatro faces do poder, que são normalmente encontradas nas organizações: coerção através da hierarquia, manipulação e integração através da definição da agenda, dominação através da formação de ideias, valores aceitos e subjetivação por meio do sentido de auto-aceitação (ALVESSON; SPICER, 2016).

O valor da metáfora para pesquisadores contemporâneos que estudam organizações é seu papel de desempenhar um papel na teorização organizacional, amplamente aceito nos dias atuais. A perspectiva de dominação trazida por Gareth Morgan (2006) em sua imagem de organizações como instrumentos de dominação, pode realmente ser usado para reexaminar as pesquisas atuais sobre organizações e o meio ambiente (JERMIER; FORBES, 2016).

Em uma revisão realizada pelo próprio Morgan (2012), a chave da discussão é se concentrar em metáforas que abordam especificamente a relação entre organizações e do ambiente natural. As metáforas das "organizações como instrumentos de dominação", buscam atribuir efeitos a consequências não intencionais, em que claramente têm um papel de jogar com as pessoas, destacando os aspectos exploradores e destrutivos.

Para propor uma discussão com acréscimo de outros autores que abordaram o tema da dominação nas organizações, este trabalho tem o objetivo de analisar sob a perspectiva de revisão sistemática, formação de redes e mapas conceituais, com adendo de um checklist de convergência, as relações entre a obra de Morgan (Capítulo 9 - A Face Repulsiva: As Organizações como Instrumentos de Dominação) e artigos selecionadas com o tema dominação, utilizando a base Scopus, trazendo as características e termos predominantes dos trabalhos e buscando uma reflexão final acerca das descobertas.

\section{REFERENCIAL TEÓRICO}

A seção teórica trata do tema da dominação pela ótica da obra de Morgan e na sequência autores que abordam o tema por diferentes prismas referenciando o capítulo 9 do livro Imagens da Organização.

\subsection{ORGANIZAÇÕES VISTAS COMO INSTRUMENTOS DE DOMINAÇÃO - OBRA DE GARETH MORGAN}

Dominação, que tem como definição domínio, subjugação, o ato de ter domínio sobre algo ou alguém, vem, ao longo da história empresarial, sendo pertinente ao domínio de grupos sociais com a prescrição 
de um anseio alheio. Unificando os conceitos de Marx (1867) com o termo dominação nas organizações, ele corrobora que a fantasma do capitalista é a alma do capital, pondera que o próprio capital tem seu acometimento para sobreviver, de criar mais-valia e de concentrar com sua parte devotada, com os meios de produção a maior quantidade aceitável de trabalho.

Na maioria das vezes, quando estas questões são tratadas na teoria organizacional, as mesmas são vistas como infortúnios, ou então como efeitos colaterais não intencionais, ou ainda como questões ligadas à ética da organização e ao relacionamento entre está e a sociedade. Ao considerar a metáfora da dominação como uma estrutura básica para a análise organizacional, a discussão deste capítulo tenta colocar estas questões na via principal, no sentido de que devem ser tratadas como dominantes nas colocações sobre a natureza e sucesso das organizações na sociedade moderna (MORGAN, 2006).

A dominação racional-legal há, em sua particularidade, a racionalidade e a burocracia. Racionalidade, é a lógica que move a sociedade moderna, lógica que fica cada vez mais condicionada de uma gerência, por leis, gastos e benfeitorias. Desta maneira, ainda sob o olhar de Weber, a dominação "é a possibilidade de impor ao comportamento de terceiros a vontade própria" (WEBER, 1920). Segundo Weber (1920) essas definições de poder ajusta-se a temática da dominação como menciona o autor que "toda a dominação manifesta -se e funciona como administração. Entusiasmar as condutas humanas é o mais perfeito caráter de exercer poder, sem acarretar distúrbio, e evitar o uso da força para alcançar bons efeitos, além de garantir o poder.

Por exemplo, muitas empresas, sob outros aspectos excelentes, frequentemente possuem registros bastante questionáveis no que diz respeito ao impacto que causam no ambiente, na força de trabalho das fábricas e no Terceiro Mundo. Embora tem obtido uma condição desenvolvida e admirável em termos de certos aspectos da prática gerencial interna, existe sempre um lado avesso desta excelência que quase sempre é complemente ignorado (MORGAN, 2006).

O conceito de imaginação procura desenvolver uma atividade proativa em relação ao modo pelo qual as organizações são e como elas poderiam ser. Acredito que as pessoas podem mudar as organizações e a sociedade, mesmo que a percepção e a verdade, ou as relações de poder verificadas através da história possam tornar, às vezes, a mudança difícil. De modo prescritivo, o autor demonstra que gostaria que todos percebessem que a realidade é feita e não dada; reconhecer que ver e compreender o mundo é sempre um ver como, em ver de um ver como sendo; e levar em conta uma ética e uma responsabilidade social em relação às consequências pessoais e coletivas do modo pelo qual vemos e agimos na vida quotidiana, difícil como isto possa ser (MORGAN, 2006). 


\subsection{DOMINAÇÃO NAS ORGANIZAÇÕES}

Na visão de Jermier e Forbes (2016) a visão de dominação das organizações, surge por estas serem profundamente divididas e politizadas ao longo das classes, ocupação, raça, etnia, gênero, meio ambiente e outras formas, acaba gerando: campos de batalha, repletos de conflitos e com resistência a práticas dominantes e exploradoras. Como um contrapeso para estudos organizacionais tradicionais que às vezes evocam pensamentos positivistas de equipes ou famílias felizes, a imagem dos campos de batalha molda a organização como um terreno disputado no qual as lutas arraigadas podem surgir de antagonismos entre pessoas com visões diferentes de questões e problemas.

Leflaive (1996) argumenta que as organizações são melhor retratadas como estruturas de dominação, onde poder e dominação se referem a uma capacidade coletiva de agir. São realizações frágeis e transitórias, concentrando momentaneamente recursos para a ação coletiva. Como tal, eles excluem e divulgam, assim fornecendo os meios para sua continuação e potencial transformação. Estudos organizacionais, empíricos ou abstratos, que pressupõem uma rigidez, ou uma estrutura de relações políticas, são suspeitos: eles não conseguem levar em consideração a fundação contínua de organizações - que é essencial para a sua relevância pragmática e teórica - e são incapazes de maneira crítica revelar a dinâmica oculta que as organizações têm para operar e mascarar essas bases dominantes.

O campo do gênero traz o argumento de que o domínio histórico dos homens na esfera pública e, em particular, quando se trata do campo de gestão e dominação da vida organizacional, levou a uma fusão de padrões tradicionais "masculinos", suposições e regras que tornam todo o campo da gestão em si um pouco alheio a muitas mulheres. Portanto, a principal opção não é tentar integrar as mulheres na esfera pública e nas posições mais altas, mas criar programas que busquem oportunidades iguais e ação afirmativa, porque acordos institucionais existentes são eles mesmos fundamentalmente falho (ALVESSON; BILING, 1992).

No nível ideológico, o sucesso da dominação se deve a legitimação do estado atual das relações de força entre as empresas que operam no capitalismo avançado, seja na égide de negócios ou entre as nações. O poder legitimador de seu pensamento torna uma fonte ideal a partir da qual os dominantes podem traçar argumentos e razões de natureza científica para justificar as situações de dominação das quais se beneficiam. No nível operacional, a dominação alcança o sucesso, devido aos conceitos que oferecem facilidade de compreensão, facilidade de implementação e posterior gratificação dos sucessos operacionais iniciais (AKTOUF; CHENOUFI; HOLFORD, 2005). 


\section{PROCEDIMENTOS METODOLÓGICOS}

A abordagem metodológica utilizada foi de pesquisa exploratória, de caráter qualitativo por meio do método da revisão sistemática, e mineração de dados textuais com o apoio do software SOBEK. A construção da pesquisa exploratória tem como objetivo conhecer as características de um fenômeno para posteriormente buscar explicações de suas causas e consequências 0 presente artigo apresenta sua construção metodológica por meio do espectro qualitativo, que em comparação ao quantitativo, é mais subjetivo e envolve a análise e reflexão das percepções para obter um entendimento de atividades sociais e humanas (RICHARDSON, 2008; COLLIS; HUSSEY, 2005).

\subsection{REVISÃO SISTEMÁTICA}

De acordo com Tranfield, Denyer e Smart (2003) os estudos de revisão sistemática podem auxiliar os estudos de gestão contribuindo principalmente com a melhoria do rigor metodológico das pesquisas. A revisão sistemática pode ser considerada como uma investigação em si mesma com caráter retrospectivo. Este tipo de investigação científica reúne, avalia criticamente e conduz a síntese dos resultados advindos de múltiplos estudos primários (CROSSAN; APAYDIN, 2010).

O método da revisão sistemática se esmera em superar possiveis direções, ao seguir um método rigoroso de busca e seleção de pesquisas, além de avaliar a relevância e a validade das informações encontradas e de coletar, sintetizar e interpretar dados. Pode contribuir na redução de erros, ao legitimar e fornecer resultados confiáveis na interpretação dos dados de um estudo (BECHEIKH; LANDRY; AMARA, 2006; KEPES; BANKS; OH, 2014).

Para a construção da análise apoiada na revisão sistemática, a base de periódicos escolhidos foi a base de dados Scopus, o critério de busca realizado trouxe o termo de maior relevância do capítulo 9 da obra "Imagens da Organização" do autor Gareth Morgan, que trata das Organizações como Instrumento de Dominação. O termo "Domination" aplicado a seleção primária com filtro por "Article title, Abstract, Keywords", se une a com o operador booleano "AND" com o título do livro "Images of Organizations" com filtro por "References", sem limites de data, acessos e tipos de publicações. Onde se apresentou os 23 artigos mais citados sobre o assunto dominação. No quadro 1 está referenciado os 23 artigos mais citados na base Scopus sobre o termo Dominação. 


\section{Gestãoe \\ Desenvolvimento}

e-ISSN: 2446-6875

p-ISSN: 1807-5436

Quadro 1: Artigos abordados na revisão sistemática

Continua

\begin{tabular}{|c|c|c|}
\hline Título & Autor e Fonte & Citações \\
\hline $\begin{array}{l}\text { Has Management Studies Lost Its } \\
\text { Way? Ideas for More Imaginative and } \\
\text { Innovative Research }\end{array}$ & $\begin{array}{l}\text { (ALVESSON, M., SANDBERG, J.) Journal of Management } \\
\text { Studies, v. } 50 \text { n. 1, p. } 128-152,2013 .\end{array}$ & 152 \\
\hline $\begin{array}{l}\text { Gender and Organization: Towards a } \\
\text { Differentiated Understanding }\end{array}$ & $\begin{array}{l}\text { (ALVESSON, M., BILLING, Y.D.) Organization Studies, v. } 13 \text { n. } \\
\text { 1, p. 73-103, 1992. }\end{array}$ & 88 \\
\hline $\begin{array}{l}\text { Power, Machines and Social Relations: } \\
\text { Delegating to Information Technology } \\
\text { in the National Health Service }\end{array}$ & (BLOOMFIELD, B.P.) Organization, v. 2, p. 489-518, 1995. & 45 \\
\hline $\begin{array}{l}\text { Perspective: An analysis of } 22 \text { years of } \\
\text { research in JPIM }\end{array}$ & $\begin{array}{l}\text { (GUO, L.) Journal of Product Innovation Management, v, 25, n. } \\
\text { 3, p. 249-260, } 2008 .\end{array}$ & 37 \\
\hline $\begin{array}{l}\text { Reflections on images of organization } \\
\text { and its implications for organization } \\
\text { and environment }\end{array}$ & $\begin{array}{l}\text { (MORGAN, G.) Organization and Environment, v. 24, n. 4, p. } \\
459-478,2011 .\end{array}$ & 23 \\
\hline $\begin{array}{l}\text { Organizing for powerlessness a critical } \\
\text { perspective on psychodynamics and } \\
\text { dysfunctionality }\end{array}$ & $\begin{array}{l}\text { (KERSTEN, A.) Journal of Organizational Change Management, } \\
\text { v. } 14, \text { n. } 5, \text { p. } 452-467,2001 .\end{array}$ & 20 \\
\hline $\begin{array}{l}\text { (Un)Conditional surrender? Why do } \\
\text { professionals willingly comply with } \\
\text { managerialism }\end{array}$ & $\begin{array}{l}\text { (ALVESSON M., SPICER, A.) Journal of Organizational Change } \\
\text { Management, v. 29, n. 1, p. 29-45, } 2016 .\end{array}$ & 19 \\
\hline $\begin{array}{l}\text { From Domination to Partnership: } \\
\text { The Hidden Subtext for Sustainable } \\
\text { Change }\end{array}$ & $\begin{array}{l}\text { (EISLER, R.) Journal of Organizational Change Management, v. } \\
7, \text { n. 4, p. } 32-46,1994 .\end{array}$ & 13 \\
\hline What have we forgotten -and why? & $\begin{array}{l}\text { (KWIATKOWSKI, R., DUNCAN, D.C., SHIMMIN, S.) Journal of } \\
\text { Occupational and Organizational Psychology, v. 79, n. 2, p. } \\
\text { 183-201, } 2006 .\end{array}$ & 10 \\
\hline $\begin{array}{l}\text { The false expectations of Michael } \\
\text { Porter's strategic management } \\
\text { framework }\end{array}$ & $\begin{array}{l}\text { (AKTOUF, O., CHENOUFI, M., HOLFORD, W.D.) Problems and } \\
\text { Perspectives in Management, v. 3, n. 4, p. 181-200, } 2005 .\end{array}$ & 10 \\
\hline $\begin{array}{l}\text { Sensemaking, Sense-Censoring and } \\
\text { Strategic Inaction: The Discursive } \\
\text { Enactment of Power and Politics in a } \\
\text { Multinational Corporation }\end{array}$ & $\begin{array}{l}\text { (WHITTLE, A., MUELLER, F., GILCHRIST, A., LENNEY, P.) } \\
\text { Organization Studies, v. 37, n. 9, p. 1323-1351, } 2016 .\end{array}$ & 9 \\
\hline
\end{tabular}




\section{Gestãoe \\ Desenvolvimento}

e-ISSN: 2446-6875

p-ISSN: 1807-5436

Continuação

\begin{tabular}{|c|c|c|}
\hline $\begin{array}{l}\text { The leadership-management } \\
\text { distinction: The domination and } \\
\text { displacement of mechanistic and } \\
\text { organismic theories }\end{array}$ & $\begin{array}{l}\text { (TERRY, L.D.) The Leadership Quarterly, v. 6, n. 4, p. 515-527, } \\
1995 .\end{array}$ & 8 \\
\hline $\begin{array}{l}\text { The failure of 'control' in the hospitality } \\
\text { industry }\end{array}$ & $\begin{array}{l}\text { (PEACOCK, M., KÜBLER, M.) International Journal of Hospitality } \\
\text { Management, v. 20, n. 4, p. 353-365, } 2001 .\end{array}$ & 7 \\
\hline $\begin{array}{l}\text { Metaphors, organizations and } \\
\text { water: Generating new images for } \\
\text { environmental sustainability }\end{array}$ & $\begin{array}{l}\text { (JERMIER, J.M., FORBES, L.C.) Human Relations, v. 69, n. 4, p. } \\
\text { 1001-1027, } 2016 .\end{array}$ & 5 \\
\hline $\begin{array}{l}\text { "Strong republic" sidetracked: } \\
\text { Oligarchic dynamics, democratization, } \\
\text { and economic }\end{array}$ & $\begin{array}{l}\text { (MANACSA, R.C., TAN, A.C.) Korea Observer, v. 43, n. 1, p. 47-87, } \\
2012 .\end{array}$ & 4 \\
\hline $\begin{array}{l}\text { Whistle While You Work? Disney } \\
\text { Animation, Organizational Readiness } \\
\text { and Gendered Subjugation }\end{array}$ & $\begin{array}{l}\text { (GRIFFIN, M., HARDING, N., LEARMONTH, M.) Organization } \\
\text { Studies, v. 38, n. 7, p. 869-894, } 2017 .\end{array}$ & 3 \\
\hline $\begin{array}{l}\text { Empowerment as Contested Terrain: } \\
\text { Employability of the Dutch workforce }\end{array}$ & $\begin{array}{l}\text { (PRUIJT, H., YERKES, M.A.) European Societies, v. 16, n. 1, p. 48- } \\
67,2014 .\end{array}$ & 3 \\
\hline $\begin{array}{l}\text { Opening and closing the door to } \\
\text { diversity: A dialectical analysis of the } \\
\text { social production of diversity }\end{array}$ & $\begin{array}{l}\text { (OMANOVIĆ, V.) Scandinavian Journal of Management, v. 29, n. } \\
\text { 1, p. 87-103, } 2013 .\end{array}$ & 3 \\
\hline $\begin{array}{l}\text { Metaphors of code-Structuring and } \\
\text { broadening the discussion on teaching } \\
\text { children to code }\end{array}$ & $\begin{array}{l}\text { (DUFVA, T., DUFVA, M.) Thinking Skills and Creativity, v. 22, p. } \\
97-110,2016 .\end{array}$ & 2 \\
\hline $\begin{array}{l}\text { Knowledge production: Public } \\
\text { management and the market } \\
\text { spectacle }\end{array}$ & $\begin{array}{l}\text { (GARRETT, T.M., SEMENTELLI, A.) International Journal of Social } \\
\text { Economics, v. 39, n. 7, p. 456-473, } 2012 .\end{array}$ & 2 \\
\hline $\begin{array}{l}\text { From virtual organization to } \\
\text { e-business: Transformational } \\
\text { structuration }\end{array}$ & $\begin{array}{l}\text { (LEE, J.J., JAYATILAKA, B., KIM, B.B., KATERATTANAKUL, P., HONG, } \\
\text { S.) International Journal of e-Business Research, v. 6, n. 4, p. } \\
\text { 13-25, } 2010 .\end{array}$ & 2 \\
\hline $\begin{array}{l}\text { In the shadow of death: Robert } \\
\text { Denhardt's Theology of Organizational } \\
\text { Life }\end{array}$ & $\begin{array}{l}\text { (KRAMER, R.) Administration \& Society, v. 21, n. 3, p. 357-379, } \\
1989 .\end{array}$ & 2 \\
\hline $\begin{array}{l}\text { A systemic approach to processes of } \\
\text { power in learning organizations: Part } \\
\text { I - literature, theory, and methodology } \\
\text { of triple loop learning }\end{array}$ & $\begin{array}{l}\text { (FLOOD, R.L., ROMM, N.R.A.) Learning Organization, v. 25, n. 4, } \\
\text { p. 260-272, } 2018 .\end{array}$ & 1 \\
\hline
\end{tabular}

Fonte: Elaborado pelos autores (2019). 
O jornal com mais relevância é o Journal of Management Studies com um fator de impacto $h$ de 145. Os fatores de impacto das revistas foram analisados e ranqueados de acordo com o Scimago Journal \& Country Rank.

\subsection{REDE DE CONCEITOS GERADO PELO SOFTWARE SOBEK MINING}

Segundo Schenker (2003) o software SOBEK Mining tem como função principal construir uma rede de conceitos a partir de um texto gramatical. A ferramenta emprega um algoritmo que consegue um diagnóstico estatístico das terminações presentes no texto e os nomeia a partir do valor integral de sua ocorrência. Esse padrão de mineração textual, designado de $n$-simple distance, pondera também as relações de proximidade entre os elementos do texto, ligando cada termo estatisticamente acentuado a N subsequentes palavras também relevantes.

Os dados gerados a partir dessa análise são expostos em uma composição organizadora do tipo grafo. Na versão do SOBEK Mining empregada na pesquisa, após a mineração do texto, o software realiza uma busca na internet por imagens associadas a cada um dos termos escolhidos e as proporciona no grafo, junto ao termo correspondente. Os grafos gerados no SOBEK Mining são editáveis, de maneira que é possivel excluir ou inserir nós, alterar as relações entre os nós e modificar as imagens relacionadas a cada termo.

No estudo realizado por Barbosa et al (2009, p. 9), utilizando a ferramenta SOBEK Mininge Tag Crowd, os autores concluíram "que ainda não há uma ferramenta completa, e o ideal é que o usuário leia sobre as características de cada uma e opte por sugerir aquela que está mais de acordo com os seus desígnios ou necessidades". O objetivo do auxílio desta ferramenta, foi de analisar as publicações, abordando o tema de dominação referenciado pela obra "Imagens da Organização" do autor Gareth Morgan, bem como a revisão sistemática do presente estudo. O emprego do software SOBEK como instrumento de aprendizagem foi proposto e analisado em estudos anteriores, como no trabalho de Reategui, Klemann e Finco (2012), que examinou seu uso como apoio à sumarização de textos.

\subsection{CONSTRUÇÃO DE MAPA CONCEITUAL DO ESTUDO}

Os mapas conceituais podem auxiliar o pesquisador no planejamento de conteúdos consisti em uma forma de opção para propagar novas ideias e quando há a necessidade de "incrementar" uma forma conceitual através de uma figura. O mapa conceitual foi desenvolvido por Joseph Novak, e se distingue por um instrumento para instituir e conceber o conhecimento (NOVAK, 1977). De acordo com Ausubel (1968) são empregues como uma elocução para definição e comunicação de conceitos e seus relacionamentos, 
e foram originalmente desenvolvidos para o suporte à aprendizagem. Nesta etapa para gerar o mapa conceitual utilizou-se o software Mindomo.

\section{APRESENTAÇÃO E ANÁLISE DOS RESULTADOS}

Esta seção visa apresentar as análises recorrentes das revisões propostas no artigo, primeiramente a sistemática, com os 23 artigos com citação registradas na base Scopus, e em segundo lugar a mineração dos dados textuais, com o mapeamento destes artigos com a utilização do software SOBEK Mining. Em terceiro foi elaborado mapa conceitual do estudo com software Mindomo na forma de uma figura conceitual apontando as relações e categorizações dos 23 artigos da revisão sistemática com o autor Morgan (2006).

\subsection{REVISÃO SISTEMÁTICA DOS ARTIGOS SELECIONADOS}

Com a definição do método de revisão sistemática definido, aplicou-se a execução da busca pelos artigos com os critérios de busca pré-estabelecidos no capítulo que destaca a metodologia. Após escolha das palavras-chave e o refinamento de critérios inclusivos, permaneceram 23 artigos, com número mínimo de citações, que serão expostos e comentados ao longo da seção. A lista de artigos resumidos é possível de ser visualizada no Apêndice A deste artigo.

Os dois artigos que encabeçam a lista, tem a participação do autor Mats Alvesson, que buscou utilizar a referência da obra de Morgan para dissertar sobre a total dominância da pesquisa incremental, com a identificação de três impulsionadores principais, condições institucionais, normas profissionais e construção de identidade. No segundo documento, é tratada a questão de gênero como uma metáfora para iluminar o caráter organizacional (ALVESSON; SANDBERG, 2013; ALVESSON, BILLING, 1992).

A abordagem da dominação tecnológica se faz presente no trabalho de Bloomfield (1995) que traz o caráter heterogêneo do tecido da vida organizacional, entrelaçando seres humanos e não-humanos e a definição de poder e dominação na relação tecnológica no interior das organizações. A utilização de metáforas no que tange máquina, organismo e cérebro, sugerindo diferentes formas pelas quais a tecnologia da informação pode ser ensinada nas escolas, com a identificação das visões dominantes e as tensões entre as visões (DUFVA; DUFVA, 2016).

O próprio autor de "Imagens da Organização" Gareth Morgan, escreveu um artigo revisando as contribuições de sua obra em pesquisas estruturadas, com foco em instrumentos de dominação, discorrendo os trabalhos desenvolvidos nos últimos 25 anos (MORGAN, 2012). 
Na sequência dos artigos revisados, existe a exploração do viés psicológico em organizações, trazendo perspectivas críticas, renúncia a autonomia em detrimento do gerencialismo, além da ênfase em estudos sobre psicologia industrial, ocupacional e organizacional, deixando clara a influência dos aspectos de mentalidade nas relações profissionais (KERSTEN, 2001; ALVESSON; SPICER, 2016; KWIATKOWSKI; DUNCAN; SHIMMIN, 2006).

Também se destacam os artigos com foco em liderança, em que os autores discutem a contribuição do conhecimento de poder e política em organizações internacionais, com foco na compreensão do sensemakingna relação subsidiária-matriz. Outro trabalho aborda com base no organicismo e mecanicismo, debatendo a distinção entre liderança e gerenciamento em uma perspectiva mais ampla. Um terceiro artigo explora relações da literatura sobre controle em uma realidade da indústria hospitaleira, em que a dependência do fracasso é relativizada com atividades empreendedoras não autorizadas (WHITTLE et al., 2016; TERRY, 1995; PEACOCK; KÜBLER, 2001).

\subsection{ANÁLISE DO CAPITULO 9, A FACE REPULSIVA: AS ORGANIZAÇÕES COMO INSTRUMENTOS DE DOMINAÇÃO}

Primeiramente com os dados obtidos do capitulo 9 A Face Repulsiva: As Organizações como Instrumentos de Dominação do livro Imagens da Organização, pode-se apresentar os principais conceitos de acordo com o SOBEK, através da mineração dos dados textuais, possíveis de serem visualizados na Figura 2. O SOBEKé uma ferramenta utilizada para extrair termos frequentes em documentos, encontrando os relacionamentos entre estes, servindo de apoio aos professores e pesquisadores no acompanhamento de trabalhos de escrita colaborativa (MACEDO et al., 2009). 


\section{Gestãoe \\ Desenvolvimento}

e-ISSN: 2446-6875

p-ISSN: $1807-5436$

Figura 2 - Rede de Conceitos gerada do capítulo 9 (A Face Repulsiva: As Organizações como Instrumentos de Dominação)

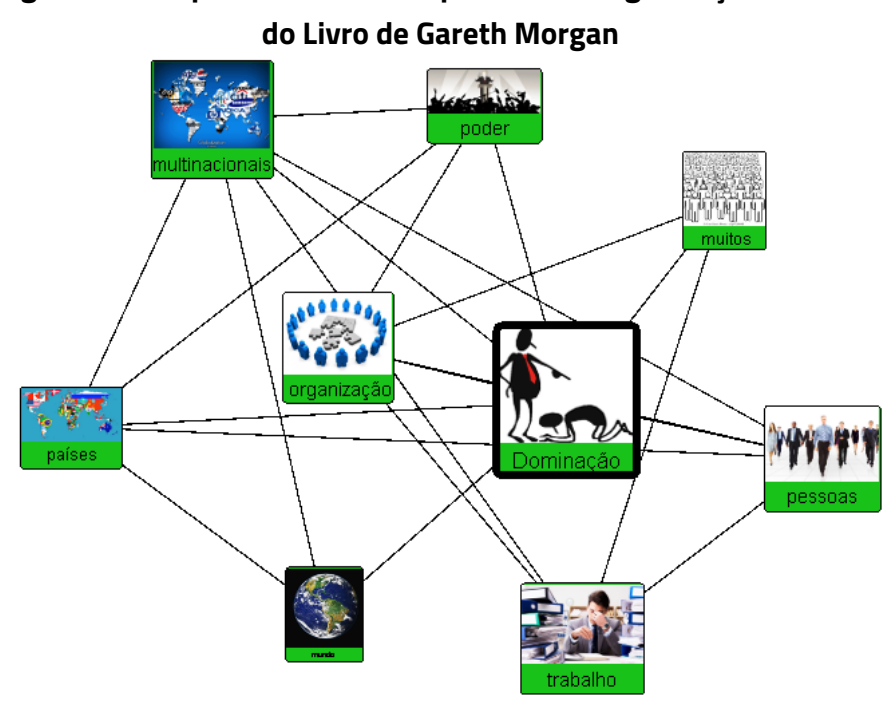

Fonte: Software SOBEK Mining (2019).

De acordo com a mineração dos dados os principais conceitos utilizados por Morgan (2006) foram, dominação, trabalho, pessoas, poder, mundo, países, organização, muitos, multinacionais, onde o software relacionou imagens a os conceitos apresentados na unidade de análise do capitulo 9, fazendo uma referência a palavra apresentada, trazendo um caráter interpretativo. Na argumentação de Flick (2009), o autor apoia que a pesquisa qualitativa abrange um caráter interpretativo e de enfoque naturalístico ante o mundo, ou seja, buscando compreender e/ou interpretar os fenômenos em termos dos sentidos que as pessoas the conferem, os pesquisadores estudam as coisas em seus argumentos naturais. Fica evidente a categoria dominação nas organizações na mineração dos dados do capitulo 9. Segundo Morgan (2006) dominação também acontece de maneiras sutis, assim como aquele que dita as normas impõe sua vontade dentro da organização sobre os outros e é visto como tendencioso o direito de cometer a dominação.

\subsection{REDE DE CONCEITOS GERADOS DOS 23 ARTIGOS DA REVISÃO SISTEMÁTICA COM O TERMO "DOMINATION"}

Em um segundo momento a partir da revisão sistemática dos 23 artigos analisados com o termo "Domination", foi possivel relacionar através da mineração dos dados dos resumos apresentados, onde se evidenciou os principais conceitos, possível de visualizar na Figura 3. 


\section{Gestãoe \\ Desenvolvimento}

e-ISSN: 2446-6875

p-ISSN: $1807-5436$

Figura 3 - Principais conceitos da revisão sistemática dos 23 artigos

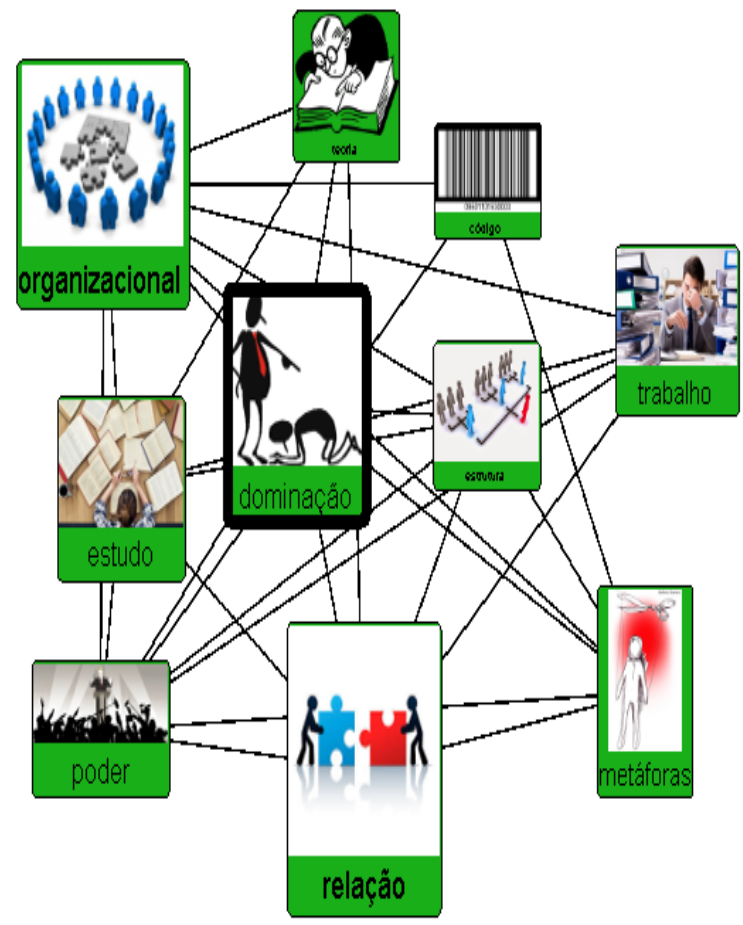

Fonte: Software SOBEK Mining (2019).

O apontamento feito pelo SOBEK dos 23 artigos da revisão sistemática apresenta os seguintes conceitos: organizacional, dominação, relação, poder, estudo, metáfora, trabalho, código, teoria, estrutura. A partir destes conceitos será feita as categorizações dos principais termos usados da revisão sistemática. Nos artigos da revisão ficou apontado a categoria dominação em evidencia, mas as a categorias organizacional e relação tem a proporção igual ou maior que a categoria dominação. A dominação nas organizações tem relações racionais apontando pontos fortes e fracos e as decisões responsáveis pelo poder pode transformar o ambiente organizacional (KRAMER, 1989; LEE et al., 2010; FLOOD;.ROMM, 2018).

\subsection{CATEGORIZAÇÃO DOS PRINCIPAIS CONCEITOS EXTRAIIDOS DO SOBEK}

Depois de efetivada a escolha dos artigos que estabelecem o corpus de análise e o capítulo 9 do livro Imagens das Organizações, foi concretizada uma leitura flutuante com vistas à categorização, ou seja, a classificação de dados obrigatórios de um conjunto por distinção, sendo estes reagrupados em seguida de acordo com a codificação. Estes reagrupamentos ocorrem de acordo com as particularidades que são 


\section{Gestãoe \\ Desenvolvimento}

e-ISSN: 2446-6875

p-ISSN: 1807-5436

comuns a estes elementos (BARDIN, 2009). Para o reagrupamento, foi feita uma terceira análise no SOBEK com conteúdo textual do capitulo 9, e a adição dos resumos feitos por meio da revisão sistemática deste estudo. A Figura 4 apresenta as principais categorizações feitas através da mineração de dados textuais.

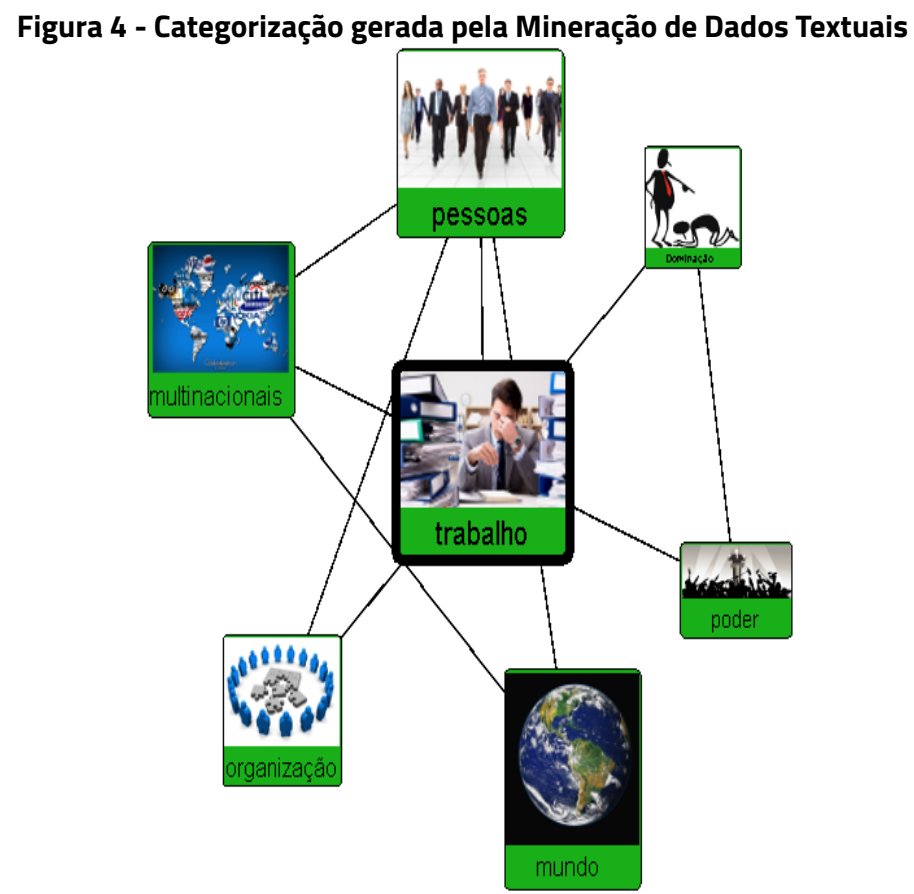

Fonte: Software SOBEK Mining (2019).

As principais categorias apresentadas foram: trabalho, pessoas, mundo, multinacionais, organização, poder, dominação. A partir dessa categorização, foi realizado um check-list nos artigos que constituem o corpus deste estudo e do capitulo 9, com a finalidade de verificar se os autores da revisão sistemática têm pontos de convergência com autor Gareth Morgan (2006) de acordo com as categorias apresentadas. Fica evidenciado de forma mais latente a categoria trabalho, e apresenta uma relação mais evidente com as categorias pessoas. $O$ estudo de Kersten (2001) fala sobre o ponto de vista de julgamento sobre organizações e a psicanálise, argumenta que a psicanálise pode dar um aporte importante tanto para o reconhecimento como para a restauração das pessoas como indivíduo na organização. 


\section{Gestãoe \\ Desenvolvimento}

e-ISSN: 2446-6875

p-ISSN: $1807-5436$

O Quadro 2 apresenta um checklist resumido dos pontos de convergência entre os autores do presente estudo.

\begin{tabular}{|c|c|c|c|c|c|c|c|c|}
\hline \multicolumn{2}{|r|}{ Categorias } & Trabalho & Pessoas & Mundo & Multinacionais & Organização & Poder & Dominação \\
\hline \multicolumn{2}{|r|}{ Artigos } & \multicolumn{7}{|c|}{ MORGAM (2006). Cap. 9 - A Face Repulsiva: AS Organizações como Instrumentos de Dominação } \\
\hline 1 & $\begin{array}{l}\text { ALVESSON, M., SANDBERG, } \\
\text { J. (2013) }\end{array}$ & $\Delta$ & $\Delta$ & & & & & $\Delta$ \\
\hline 2 & $\begin{array}{l}\text { ALVESSON, M., SANDBERG, } \\
\text { J. (1992) } \\
\end{array}$ & $\boldsymbol{D}$ & $\boldsymbol{D}$ & & & 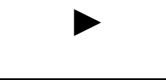 & & \\
\hline 3 & BLOOMFIELD, B.P. (1995) & & & & & D & 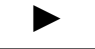 & $\boldsymbol{D}$ \\
\hline 4 & GUO, L (2008) & & & $>$ & & & & \\
\hline 5 & MORGAN, G (2011) & 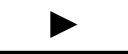 & 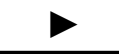 & 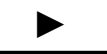 & $\nabla$ & $>$ & $\nabla$ & $\nabla$ \\
\hline 6 & $\begin{array}{l}\text { KERSTEN, A } \\
(2001)\end{array}$ & & 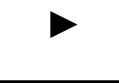 & & & 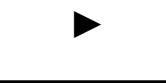 & & \\
\hline 7 & $\begin{array}{l}\text { ALVESSON M., SPICER, A. } \\
(2016)\end{array}$ & 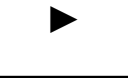 & $\Delta$ & & & 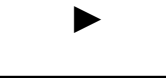 & & $\Delta$ \\
\hline 8 & EISLER, R. (1994) & 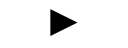 & & & & $>$ & & \\
\hline 9 & $\begin{array}{l}\text { KWIATKOWSKI, R., DUNCAN, } \\
\text { D.C., SHIMMIN, S. (2006) } \\
\end{array}$ & & 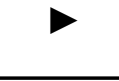 & 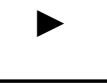 & & 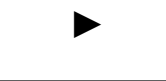 & $>$ & \\
\hline 10 & $\begin{array}{l}\text { AKTOUF, O., CHENOUFI, } \\
\text { M., HOLFORD, W.D. (2005) }\end{array}$ & 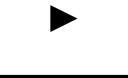 & $>$ & & & $\Delta$ & & \\
\hline 11 & $\begin{array}{l}\text { WHITTLE, A., MUELLER, F., } \\
\text { GILCHRIST, A., LENNEY, P. } \\
\text { (2016) }\end{array}$ & 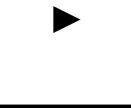 & $\boldsymbol{D}$ & $>$ & & & $\nabla$ & \\
\hline 12 & TERRY, L.D (1995) & & D & & & 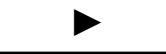 & & \\
\hline 13 & $\begin{array}{l}\text { PEACOCK, M., KÜBLER, M. } \\
\text { (2001) }\end{array}$ & 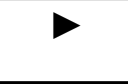 & & & & $>$ & $\nabla$ & \\
\hline 14 & $\begin{array}{l}\text { JERMIER, J.M., FORBES, L.C. } \\
\text { (2016) }\end{array}$ & & 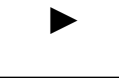 & & & 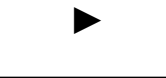 & & \\
\hline 15 & $\begin{array}{l}\text { MANACSA, R.C., TAN, A.C. } \\
\text { (2012) }\end{array}$ & & & $>$ & & & 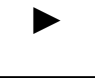 & 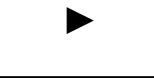 \\
\hline 16 & $\begin{array}{l}\text { GRIFFIN, M., HARDING, } \\
\text { N., LEARMONTH, M. (2017) }\end{array}$ & 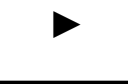 & 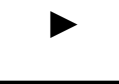 & & $\nabla$ & $\nabla$ & $>$ & 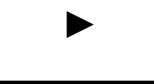 \\
\hline 17 & $\begin{array}{l}\text { PRUIJT, H., YERKES, M.A. } \\
(2014)\end{array}$ & $>$ & & 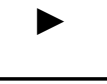 & & $>$ & $>$ & $>$ \\
\hline 18 & OMANOVIĆ, V (2013) & $\nabla$ & & & 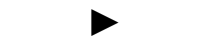 & $>$ & 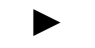 & \\
\hline 19 & DUFVA, T., DUFVA, M. (2016) & $>$ & 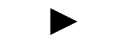 & & & & $>$ & $>$ \\
\hline 20 & $\begin{array}{l}\text { GARRETT, T.M., SEMENTEL- } \\
\text { LI, A. (2012) }\end{array}$ & & & 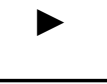 & & $\nabla$ & 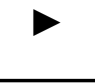 & 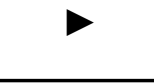 \\
\hline 21 & LEE, J.J. et al, (2010) & & & & & 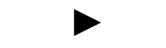 & & $>$ \\
\hline 22 & KRAMER, R. (1989) & & & & & $>$ & & 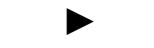 \\
\hline 23 & $\begin{array}{l}\text { FLOOD, R.L., ROMM, N.R.A. } \\
\text { (2018) }\end{array}$ & & & 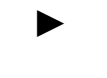 & & $>$ & & $>$ \\
\hline
\end{tabular}

Fonte: Elaborado pelos autores (2019). 
A partir da análise do Quadro 2, alguns fatos chamam a atenção. Fica latente que vinte e dois artigos abordam a categoria organização e tem ligação com o capitulo 9, doze artigos abordam a categoria trabalho e apresentam relação com a categoria organização ambas ligadas com o capitulo estudado neste estudo. Doze artigos foram apontados na categoria pessoas e tem relação com o capitulo 9, a categoria organização tem uma relação com a categoria pessoas e a categoria trabalho. Outros doze artigos foram categorizados como dominação, onze artigos foram categorizados como poder, sete artigos foram categorizados como mundo, três artigos categorizados com multinacionais. Todos os artigos têm relação com o autor Morgan (2006).

\section{CONSIDERAÇÕES FINAIS}

O tema da dominação no ambiente organizacional vista pela ótica de Morgan e os autores destacados neste documento, mostram um grau de convergência destacado, pois 18 dos 23 artigos selecionas, utiliza alguma referência da metáfora de dominação em suas construções de pesquisa. A exploração de diferentes visões sobre a dominação, traz à tona como este pode ser um assunto multifacetado, pois é possível observar nas publicações, discussões sobre o tema em perspectiva psicológica, de gênero, tecnológica, liderança e até mesmo no campo da pesquisa acadêmica.

A formação das redes conceituais apresentado no Apêndice " $\mathrm{A}$ ", apresenta claramente que quando isolamos Morgan e os autores dos artigos selecionados em figuras diferentes, a dominação prepondera como o termo central das obras, mas com diferentes pontos de vista e proposição de tamanho das discussões. Enquanto Morgan se concentrou em aplicar sua metáfora a magnitude de organizações globais, multinacionais, países e as pessoas como meros elementos no jogo organizacional, os 23 autores analisados em paralelo, recorreram a questões mais de superfície e com detalhes nas rotinas das organizações, como estudo, códigos morais, poder do líder, relações e estrutura.

Ao se propor o cruzamento conceitual entre Morgan e os autores, a dominação já ganha um papel secundário, com o trabalho e as pessoas tomando o centro das discussões, trazendo a mistura da questão global com o poder e as organizações. Essa impressão é corroborada no checklist de convergência entre os autores, com a predominância clara entre os trabalhos, dos termos trabalho, pessoas e organização, deixando claro que a mensagem que trabalhos destes estudos buscam transmitir, são os desafios humanos nas organizações, sejam de qual campo de atuação forem, que as pessoas são orientadas por um sistema de dominação, a lidar com este complexo tema, seja por meio de metáforas ou pesquisa aplicada. 


\section{Gestãoe \\ Desenvolvimento}

e-ISSN: 2446-6875

p-ISSN: $1807-5436$

Desta maneira, a percepção das dificuldades assume importantes posições no estabelecimento dos relacionamentos verticais entre as hierarquias na organização, por conseguinte a complexidade dos estudos efetuados talvez venha a ressaltar a relatividade de alternativas às soluções ortodoxas. Por outro lado, a contínua expansão de nossa atividade nos obriga a análise dos paradigmas corporativos nas organizações. De uma forma positivista pode-se apresentar que autores no ano de 2019 ainda pesquisam o termo "Dominação" nas organizações, atestando que o estudo tem uma relevância acadêmica, bem como gerencial. Como limitações da pesquisa, cabe destacar o uso somente de uma da base de dados Scopus, que restringiu o alcance do estudo. Poderia ser feita uma busca em outras bases como: Web of Science, Emerald e Science Direct. Para pesquisas futuras, outras alternativas de buscas, com os termos "Domination in public organizations", "Domination in higher education organizations" e "Domination in military organizations", cruzando com o capítulo 9 do livro Imagens das Organizações de Morgan (2006), seriam opções para diferentes abordagens e reflexões deste importante tema que permeia o ambiente organizacional.

\section{REFERÊNCIAS}

AKTOUF, O.; CHENOUFI, M.; HOLFORD, W. D. The False Expectations of Michael Porter's Strategic Management Framework. Problems and Perspectives in Management, v. 3, n. 4, p. 181-200, 2005.

AUSUBEL, D.P. Educational psychology: a cognitive view. New York, Holt, Rinehart and Winston. 1968.

ALVESSON, M.; BILLING, Y. D. Gender and Organization: Towards a Differentiated Understanding. Organization Studies, v. 13, n. 1, p. 73-103, 1992.

ALVESSON, M.; SPICER, A. (Un) Conditional surrender? Why do professionals willingly comply with managerialism? Journal of Organizational Change Management, v. 29, n. 1, p. 29-45, 2016.

BARBOSA, M. L.; SEVERO, C.; REATEGUI, E. B. Mineração de padrões no gênero textual blog. Revista Novas Tecnologias na Educação, Porto Alegre, v. 7, n³ 3, dezembro, 2009.

BARDIN, L. Análise de conteúdo. 4. ed. Paris: PUF, 2009.

BECHEIKH, N.; LANDRY, R.; AMARA, N. Lessons from innovation empirical studies in the manufacturing sector: A systematic review of the literature from 1993-2003. Technovation, v. 26, p. 644-664, 2006.

COLLIS, J.; HUSSEY, R. Pesquisa em administração: um guia prático para alunos de graduação e pós-graduação. 2. ed. Porto Alegre: Bookman, 2005. 


\section{Gestãoe \\ Desenvolvimento}

e-ISSN: 2446-6875

p-ISSN: 1807-5436

CROSSAN, M. M.; APAYDIN, M. A. Multi-Dimensional Framework of Organizational Innovation: A Systematic Review of the Literature. Journal of Management Studies, v. 47, n. 6, p. 1154-1191, 2010.

FLICK, U. Métodos de Pesquisa: introdução à pesquisa qualitativa. 3ª Ed. Porto Alegre: Artmed, 2009.

FLOOD, R. L.; ROMM, N. R. A. A systemic approach to processes of power in learning organizations: Part I - literature, theory and methodology of triple loop learnin. Learning Organization, v. 25, n. 4, p. 260$272,2018$.

JERMIER, J. M.; FORBES, L. C. Metaphors, organizations and water: Generating new images for environmental sustainability. Human Relations, v. 69, n. 4, p. 1001-1027, 2016.

KEPES, S.; BANKS, G. C.; OH, I.-S. Avoiding Bias in Publication Bias Research: The Value of "Null" Findings. Journal of Business and Psychology, v. 29, n. 2, p. 183- 203, 2014.

KERSTEN, A. Organizing for powerlessness a critical perspective on psychodynamics and dysfunctionality. Journal of Organizational Change Management, v. 14, n. 5, p. 452-467, 2001.

KRAMER, R. In the shadow of death: Robert Denhardt's Theology of Organizational Life. Administration \& Society, v. 21, n. 3, p. 357-379, 1989.

LEFLAIVE, X. Organizations as Structures of Domination. Organization Studies, v. 17, n. 1, p. 23-47, 1996.

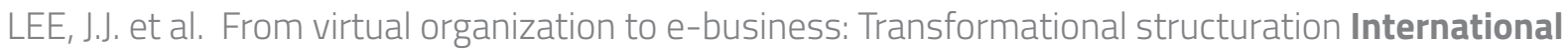
Journal of e-Business Research, v. 6, n. 4, p. 13-25, 2010.

MACEDO, A., et al. Using Text-Mining to Support the Evaluation of Texts Produced Collaboratively. Education and Technology for a Better World; Selected papers of the 9th. World Conference on Computers in Education. [s.n.], 2009.

MARX, Karl. $\mathbf{O}$ capital: crítica à economia política. Tradução de Reginaldo Santanna. Ano da publicação original: 1867. 19. ed. Rio de Janeiro: Civilização Brasileira, 2002.

MINDOMO. Software. Mapas Conceituais. Disponível em: https://www.mindomo.com/pt/. Acesso em: 15 jan. 2019.

MORGAN, Gateth. Imagens da Organização. Trad. Geni G. Goldschmidt. $2^{a}$ ed. - $4^{a}$ Reimpressão - São Paulo: Atlas, 2006. 


\section{Gestãoe \\ Desenvolvimento}

e-ISSN: 2446-6875

p-ISSN: $1807-5436$

MORGAN, G. Reflections on Images of Organization and Its Implications for Organization and Environment. Organization \& Environment, v. 24, n. 4, p. 459-478, 2012.

NOVAK, J. D. (1977). A theory of education. Ithaca, NY: Cornell University Press.

REATEGUI, E.; KLEMANN, M.; FINCO, M. D. Using a text mining tool to support text summarization. In: IEEE INTERNATIONAL CONFERENCE ON ADVANCED LEARNING TECHNOLOGIES, 12., 2012, Rome. [Proceedings...][S.I.]: IEEE, 2012. p. 607-609. Disponivel em: <https://doi.org/10.1109/ICALT.2012.51>. Acesso em: 14 jan. 2019.

RICHARDSON, R. J. Pesquisa social: métodos e técnicas. 3. ed. São Paulo: Atlas, 2008.

SOBEK. Sobek Mining. Minerador de Textos Sobek, 2015. Disponível em: <http://sobek.ufrgs.br/index. html>. Acesso em: 05 Jan 2019.

SCHENKER, A. Graph-theoretic techniques for web content mining. 2003. Thesis (Doctor of Philosophy) - Department of Computer Science and Engineering, College of Engineering, University of South Florida, 2003. Disponivel em: <http://scholarcommons.usf.edu/cgi/viewcontent.cgi?article=2466\&context=etd>. Acesso em: 13 Jan, 2019.

TRANFIELD, D.; DENYER, D.; SMART, P. Towards a Methodology for Developing Evidence-Informed Management Knowledge by Means of Systematic Review. British Journal of Management, v. 14, n. 3, p. 207-222, 2003

WEBER, Max. Economia e sociedade: fundamentos da sociologia compreensiva. Tradução de Regis Barbosa e Karen Elsabe Barbosa. Ano da publicação original: 1920. Brasília: Editora Universidade de Brasília, 1999, Reimpressão: 2009. 


\section{Gestãoe \\ Desenvolvimento}

e-ISSN: 2446-6875

p-ISSN: 1807-5436

APÊNDICE A - MAPA CONCEITUAL DA RELAÇÃO DAS CATEGORIAS DO ESTUDO E SEUS RESPECTIVOS AUTORES

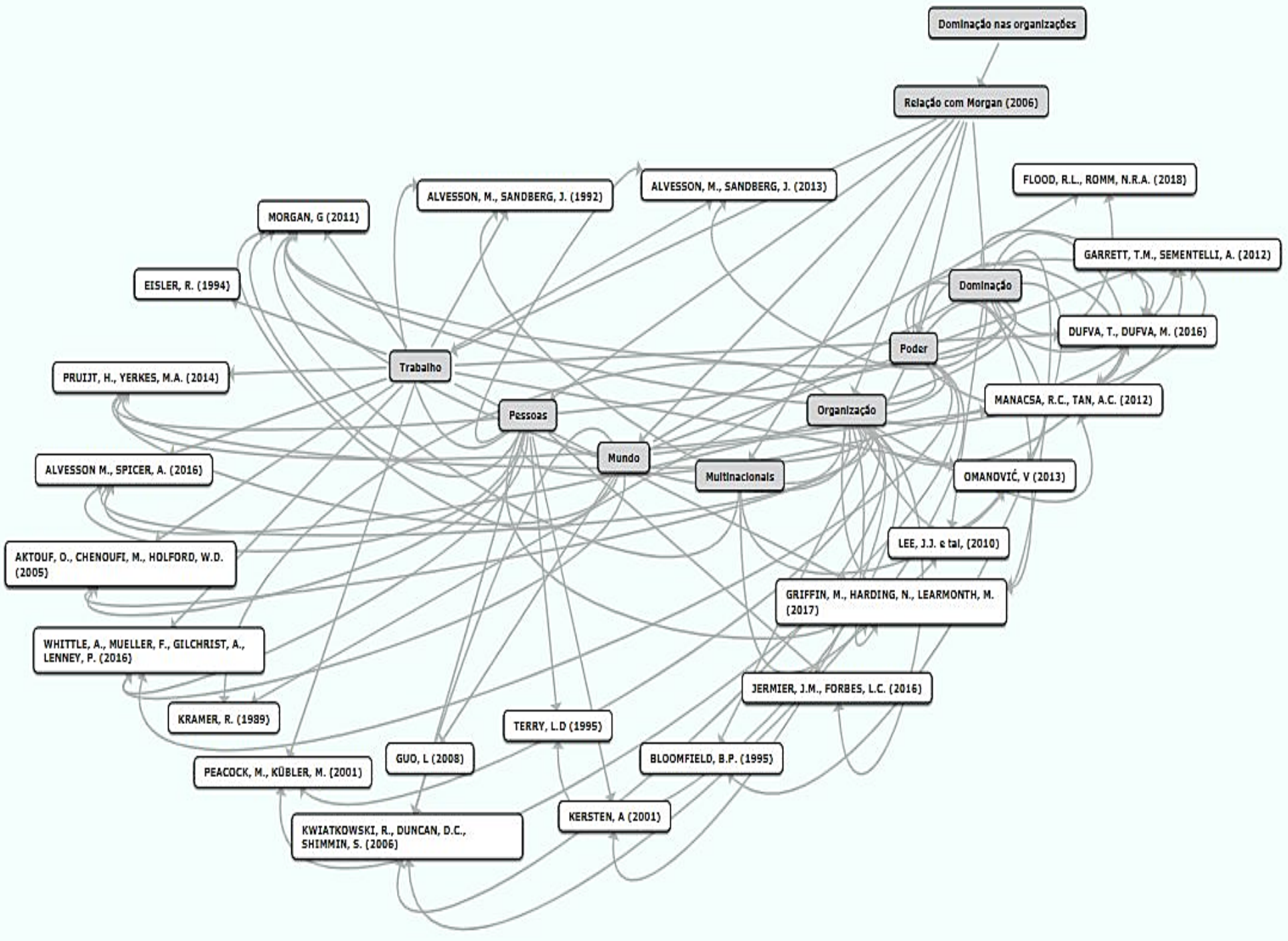

Fonte: Elaborado pelos autores (2019). 